\title{
ANÁLISE EXPLORATÓRIA ENTRE: INFECTADOS POR DENGUE E DADOS SOCIOECONÔMICOS, RELATIVOS A 2010, DOS MUNICÍPIOS DA REGIÃO SUDESTE DO BRASIL
}

\author{
Diego de Sousa Ribeiro Fonseca \\ Ricardo Alexandrino Garcia
}

\section{Resumo}

O objetivo deste trabalho foi realizar a análise exploratória de dados referentes às taxas registradas de acometidos pela dengue nos municípios dos Estados da região Sudeste do Brasil, correlacionando-os com as seguintes variáveis: índice de desenvolvimento humano municipal (IDHM), percentual de população urbana, renda per capita, esperança de vida ao nascer e população economicamente ativa (PEA) maior que 18 anos. A metodologia empregada consistiu na análise descritiva dos dados; análise de componentes principais; análise da correlação de Pearson e emprego do teste de semelhança por vizinhança baseado no Índice de Moran. Os resultados mostraram alta dispersão nos dados, tendo como modelo preditivo com maior poder explicativo a relação entre a dengue e o IDHM nos Estados. Houve correlação entre dengue e IDHM nas seguintes unidades federais: Minas Gerais, Rio de Janeiro e São Paulo. Entre dengue e percentual de população urbana: em Minas Gerais, Espírito Santo e São Paulo. Entre dengue e renda per capita: em Minas e no Rio. Entre dengue e esperança de vida ao nascer: em Minas e São Paulo. Associação entre dengue e população economicamente ativa: no Estado de Minas Gerais. A heterogeneidade dos valores foi verificada por meio da análise espacial com o İndice de Moran, o qual foi significativo $(\mathrm{p}<0.01)$, mas moderadamente explicativo $(0,50)$. A conclusão a que se chega é, quanto maior o nível de desenvolvimento, maior tem sido a possibilidade da ocorrência de dengue e, menor é a capacidade de tais sociedades em conter tal distúrbio da saúde coletiva.

Palavras-chave:infectados por dengue, variáveis socioeconômicas, análise de componentes principais, análise de correlação e Índice de Moran. 


\section{1- INTRODUÇÃO}

A dengue é uma doença transmitida pelo inseto Aedes aegypti, o qual é descrito como menor que um mosquito comum, é preto e com pequenas manchas brancas, suas asas são translúcidas e o ruído que produz não chega a ser notado pelo ouvido humano. $\mathrm{O}$ macho alimenta-se exclusivamente de frutas, já a fêmea precisa de sangue para o amadurecimento dos ovos, que são depositados nas paredes internas dos objetos, em locais úmidos e com água parada. No momento da postura são brancos, mas em pouco tempo tornam-se pretos e brilhantes. Mesmo que a água onde estão depositados seque, os ovos não morrem, e, ao primeiro contato com a umidade há a eclosão (BRAZ et al., 2013).

O entendimento do padrão de incidência da dengue é importante porque conforme a Organização Mundial da Saúde (OMS), entre 50 a 100 milhões de pessoas se infectam por dengue anualmente em mais de 100 países, de todos os continentes, exceto o europeu. Desse total, cerca de 550 mil doentes necessitam de hospitalização e 20 mil falecem (FONSECA; BRAZ, 2010; BRAZ et al., 2013). Para essa doença não há tratamento específico, existe apenas o controle dos sintomas e o trabalho dos agentes de saúde consiste na prevenção (JENTES et al., 2011).

Regis et al. (2009) discutiram que a epidemia de dengue recorrente em países como o Brasil é pautada em fatores climáticos e histórico culturais, sendo esses os responsáveis pelo seu alastramento e difícil controle. Os autores salientaram que apesar dos esforços governamentais em controlar a epidemia, não tem havido decréscimo nos índices de infestação do país, e que novas ferramentas de vigilância e análise a essa ameaça devem ser providenciadas (PIETRI; GARCÍA, 2008; PESSANHA et al., 2009; BRAZ et al., 2013).

O objetivo deste trabalho é realizar uma análise exploratória de dados, buscando evidências espaciais da ocorrência de enfermos notificados com a dengue, no ano de 2010, empregandose a correlação das taxas de ocorrência da dengue nos Estados da região Sudeste do Brasil, com as seguintes variáveis: índice de desenvolvimento humano municipal, percentual de população urbana, renda per capita, esperança de vida ao nascer e população economicamente ativa (PEA) maior que 18 anos. O uso das tais variáveis socioeconômicas permite esclarecer se os dados que apontam para o nível de desenvolvimento das sociedades estariam intrínsecos à capacidade dessas mesmas sociedades em prevenir problemas de ordem coletiva, tais como a incidência de epidemias como a dengue.

Por meio dos resultados espera-se compreender melhor o padrão de distribuição espacial da dengue a partir da associação com variáveis socioeconômicas, e, dessa forma, apontar zonas 
críticas e propícias à maior disseminação dessa epidemia que se tornou ameaça à saúde da sociedade contemporânea.

\section{2- MATERIAIS E MÉTODOS}

\section{1- Área de estudo}

A região Sudeste do Brasil (Figura 1) é aquela onde há a maior população total do país, o equivalente a 80.364 .410 habitantes, valor que representa $42,1 \%$ da população absoluta. A taxa de fecundidade é de 1,70 filhos por mulher, sendo esta, a menor taxa entre as grandes regiões brasileiras. Possui a $2^{a}$ menor taxa de mortalidade infantil, equivalente a 13,1\%o. Apresenta o maior percentual de população com nível superior completo $(10 \%)$, e, trata-se da população, com mais de 10 anos de idade, com o segundo maior rendimento salarial médio, equivalente a 1.575 reais (IBGE, 2010). É a região brasileira com o $2^{\circ}$ maior índice de desenvolvimento humano (IDH), igual a 0,824 (FJP, 2013).

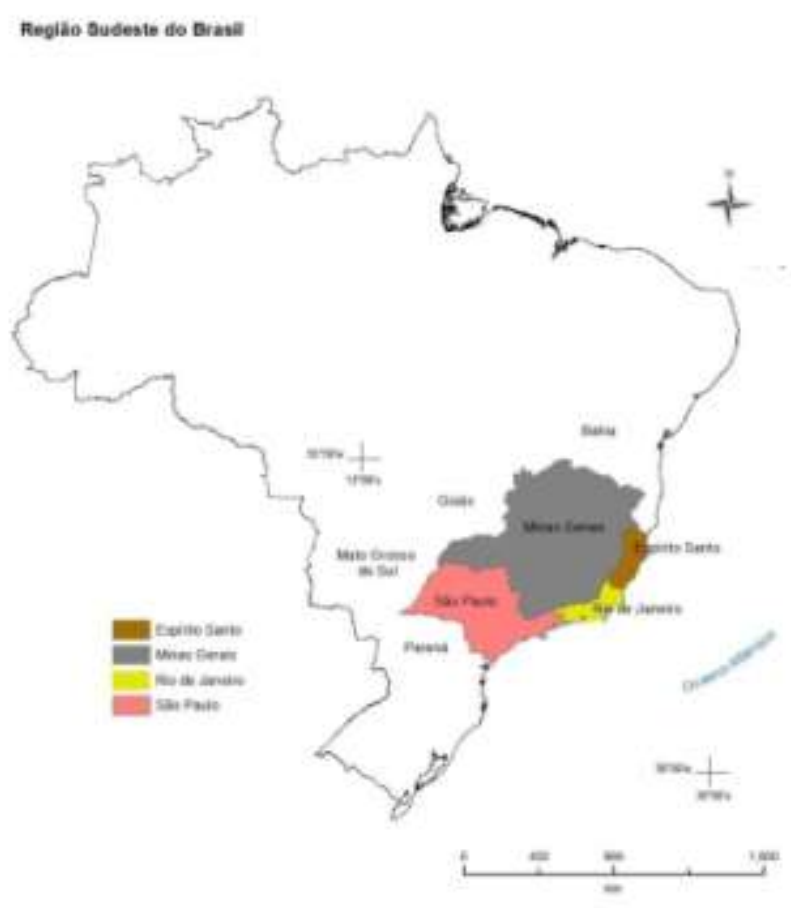

Figura 1. Localização da região Sudeste do Brasil e áreas vizinhas. 


\section{2- Procedimentos operacionais}

Para realização do trabalho foram utilizadas informações sobre a taxa de incidência de enfermos por dengue, referentes aos municípios das unidades federativas da região Sudeste do Brasil: Minas Gerais, Espírito Santo, Rio de Janeiro e São Paulo. Tais taxas foram originadas do Ministério da Saúde (MS, 2010).O cruzamento da taxa de incidência por dengue foi realizado com as seguintes variáveis: índice de desenvolvimento humano municipal (IDHM), adquirido junto a Fundação João Pinheiro (2013); porcentagem da população urbana, renda per capita, esperança de vida ao nascer, e população economicamente ativa maior de 18 anos (IBGE, 2010). O universo de análise correspondeu ao número de municípios por Estado, tendo Minas Gerais: 853; Espírito Santo: 78; Rio de Janeiro: 92; São Paulo: 645 escores.

Para análise quantitativa, foram usados os aplicativos computacionais estatísticos: o Bioestat, versão 5.3 (AYRES, 2007), e o Stata, versão 12 (Stata Corporation, College Station, Estados Unidos). Por meio do software Stata realizou-se a estatística descritiva, com a finalidade de obtenção dos valores referentes à média e ao coeficiente de variação, para a verificação da dispersão dos dados. Também foi realizado o teste de hipóteses (ANOVA) juntamente com o teste de Tukey, a fim de verificar se ocorrem diferenças significativas entre as séries numéricas e quais as médias são diferentes. Além desses testes mencionados, foi feita a correlação de Pearson, para avaliar o grau de associação das variáveis, sendo esse obviamente, um ensaio aleatório, não determinístico (MARTINS, 2010).

Com o manuseio do Bioestat realizou-se a análise de componentes principais, com objetivo de realizar a exploração dos dados e, portando, dos seus níveis de significância relativa com as variáveis preditivas, tendo em vista a quantidade de escores que as mesmas dispuseram para realização deste trabalho.

Para construção dos mapas foi usado o aplicativo computacional ArcGIS, versão 10.1, pelo qual foi processada também a análise de similaridade por vizinhança, através do Índice de Moran, onde foi usada a "banda fixa" em 15.000 metros como modelo de concepção espacial dos clusters. Os arquivos em formato shapefile, relativos à espacialização dos municípios, foram adquiridos junto ao IBGE (2015).

O Índice de Moran é um teste cuja hipótese de nulidade é a independência espacial. Neste último caso, seu valor seria zero. Valores positivos (entre 0 e +1 ) indicam para correlação direta, e negativos (entre 0 e -1) correlação inversa (CAMPOS; MACEDO, 2014). Essa aplicação serve então para averiguar se uma amostra ou população possui auto-correlação espacial em relação a uma variável específica (ANSELIN, 1995). 
A quantidade e os intervalos de classes dos mapas foram calculados conforme o modelo de Sturges, aplicado conforme o universo amostral para cada unidade da federação, tal como é visto em Martins (2010), com a fórmula:

$$
h \cong R \div K
$$

Onde:

$R=$ maior medida - menor medida $;$ $=1+3,33(\log n)$.

\section{3- RESULTADOS E DISCUSSÃO}

Ao observar a Figura 2 é visualizado que os municípios mineiros onde houve maior incidência de infectados por dengue foram, em geral, aqueles da porção centro-norte e alguns municípios discrepantes do centro-sul do Estado, seguido daqueles da parte localizada à leste dessa unidade federativa, com alta heterogeneidade nos valores, sendo portanto, difícil uma identificação espacial homogênea. Os Estados de São Paulo e Espírito Santo tiveram predominância de municípios com taxas elevadas na porção norte, e, no caso de São Paulo, também na região sul. No Estado do Rio de Janeiro houve predomínio de infectados nas cidades pertencentes aos municípios que margeiam a orla marítima, de norte a sul, nessa unidade federativa.

As áreas com as menores taxas estão localizadas, sobretudo, na região centro-sul do Estado de Minas Gerais; centro do Estado do Espírito Santo; centro-sul do Estado do Rio de Janeiro; e do centro até o início da porção sul do Estado de São Paulo. Tal como as informações aparecem na Figura 2, aparentemente há um padrão espacial de infectados pela dengue nos respectivos municípios. 


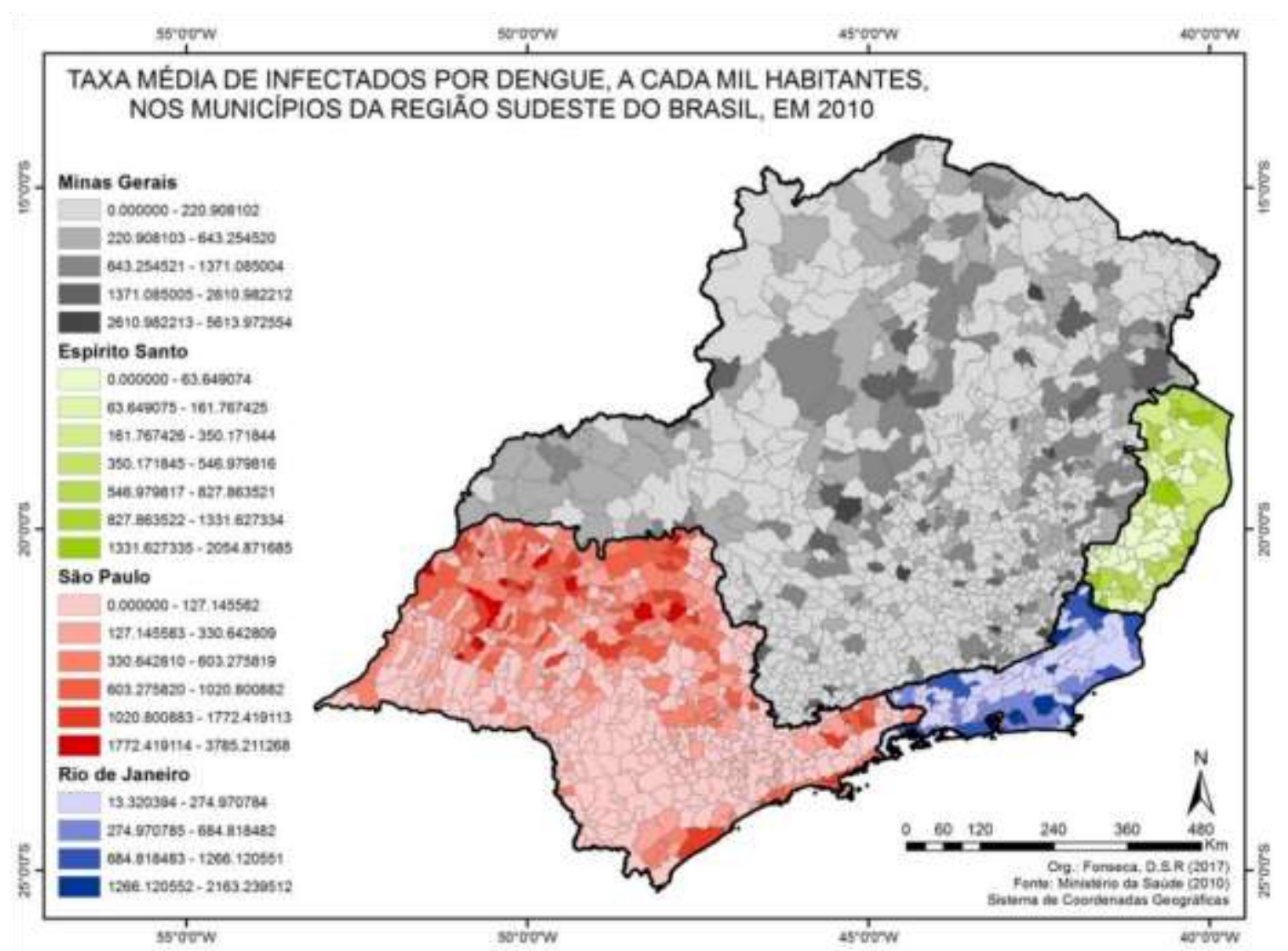

Figura 2. Taxa de infectados por dengue (2010) nos municípios dos Estados da região Sudeste do Brasil.

O teste de hipóteses empregado mostra diferenças significativas $(p<0,01)$, entre as médias de infectados por dengue em algumas das unidades federativas do Sudeste do Brasil, tal como pode ser visto na Tabela 1 . Os valores evidenciam que o número de pessoas infectadas por dengue nos Estados não tem relação direta com o tamanho da unidade federativa, tendo em vista que o Estado de Minas Gerais, aquele com maior número de municípios, apresentou a menor incidência de dengue. E, de forma similar, o Rio de Janeiro, um dos estados com menor número de municípios, apresentou a maior incidência. Os respectivos coeficientes de variação mostram alta dispersão dos valores (MARTINS, 2010). Contudo, as taxas de infestação ocorrem de forma mais homogênea nos municípios do Rio de Janeiro, quando comparadas ao Estado de Minas, onde há maior heterogeneidade. 


\section{Tabela 1}

Incidência Média de infectados por dengue e seus respectivos coeficientes de variação, por unidades Federativas da região Sudeste do Brasil, 2010.

\begin{tabular}{ccc}
\hline Unidade Federativa & Incidência Média da Dengue & Coeficiente de Variação (\%) \\
\hline Rio de Janeiro & $280.198 \mathrm{a}$ & 98,69 \\
\hline São Paulo & $204.087 \mathrm{ab}$ & 120,16 \\
\hline Espírito Santo & $183.949 \mathrm{~b}$ & 125,58 \\
\hline Minas Gerais & $159.386 \mathrm{~b}$ & 144,03 \\
\hline
\end{tabular}

Fonte: Ministério da Saúde (2010).

Nota: as médias seguidas por letras idênticas não diferem entre si pelo teste de Tukey a 1\% de significância.

Barbosa; Lourenço (2010), em pesquisa realizada no município de Tupã, Estado de São Paulo, também mostraram existir um padrão heterogêneo na distribuição de enfermos por dengue em seu local de pesquisa. Tais autores acharam ainda uma correlação significativa entre a intensidade de ocorrência larvária (Aedes aegypti) e casos de dengue. Essas informações indicam existir fatores muito intrínsecos a cada local para ocorrência de zonas críticas de propagação da doença, o que torna a análise dos fatores envolvidos, sendo eles biológicos ou socioambientais, demasiadamente complexos.

Conforme as informações disponíveis para realização deste trabalho, em Minas Gerais os municípios que apresentaram as maiores taxas de incidência da dengue no período analisado foram: Passos (5.614); Rio Novo (4.703); e Martins Soares (4.703). Cento e vinte e cinco (125) municípios, num universo de 853, apresentaram incidência igual à zero. Os municípios mineiros com as maiores taxas de dengue, valores discrepantes, estão inseridos na região centro-sul do Estado, sendo essa, a de maior ocorrência de precipitação (VIOLA et al., 2010).

Entre os capixabas, os municípios que apresentaram as taxas mais elevadas de dengue foram: Colatina (2.055); Pinheiros (1.845); e Marilândia (1.723). Os três com menor incidência, entre os 78, foram: Divino de São Lourenço $(0,0)$; Brejetuba $(8,4)$; e Irupi $(8,4)$. Os locais com maior incidência de dengue, tais como: Colatina e Marilândia, localizados no centro do Estado, e Pinheiros, posicionado ao norte do Estado, estão em regiões de ocorrência dos maiores índices de evapotranspiração e pluviosidade do Espírito Santo (CASTRO et al., 2010).

Dos municípios cariocas, os que apresentaram as taxas de incidência da dengue mais elevadas foram: Cordeiro (2.163); Bom Jesus do Itabapoana (2.154); e Tanguá (2.154). Aqueles com menor incidência, do total de 92, foram: Rio Claro (13,3); Teresópolis $(14,7)$; e Sumidouro $(20,0)$. Nos locais com as maiores taxas de dengue, entre eles: Cordeiro, localizado no centro do Estado, Bom Jesus do Itabapoana, no extremo norte, e Tanguá, ao sul - estão posicionados nas 
faixas de isoietas com elevados índices de chuva no Rio de Janeiro, principalmente no verão (SILVA; DERECZYNSKI, 2014).

Os municípios paulistas que apresentaram taxas mais elevadas da dengue foram: Nova Castilho (3.785); Potim (3.178); e Ribeirão Preto (3.149). Trinta e um (31) municípios, num universo de 645, apresentaram incidência igual à zero. Os municípios do Estado de São Paulo que apresentam a maior ocorrência da doença estudada estão distribuídos espacialmente nas isoietas com os mais elevados índices pluviométricos da região paulista (CARVALHO et al., 2012).

A maior incidência da dengue poderia estar obedecendo a um padrão sazonal muito atrelado aos índices pluviométricos do período de análise (SANTOS; MARÇAL JÚNIOR, 2004; VIEIRA; LIMA, 2006). Nesse sentido, nas pesquisas, é imprescindível a manipulação de amostras com maior amplitude de tempo e locais a fim de se promover observações mais concisas a respeito dos surtos e áreas de risco.

A realização da análise de componentes principais como modelo para análise exploratória dos dados mostrou que para o Estado de Minas Gerais, as variáveis: incidência da dengue (54,64\%), IDHM (17,93\%), e população urbana (13,91\%) - representaram 86,49\% da variação dos indivíduos testados. Os dados relativos ao Estado do Espírito Santo mostraram que as variáveis: incidência da dengue (57,53\%) e IDHM (17,64\%) - representam 75,17\% da variação dos indivíduos testados. Para o Estado de São Paulo, as variáveis: incidência da dengue (40\%), IDHM (21,31\%), população urbana $(18,06 \%)$ e renda per capita $(13,84 \%)$ - representam 93,23\% da variação dos indivíduos testados. E, para o Estado do Rio de Janeiro, as variáveis: incidência da dengue $(51,79 \%)$, IDHM (17,94\%), população urbana $(13,10 \%)$ - representam $82,83 \%$ da variação dos indivíduos testados.

Em busca de aprofundar o entendimento do padrão de distribuição da dengue na região analisada e, dessa forma, apontar as áreas mais propícias à ocorrência de surtos da doença, realizou-se correlações entre taxas de incidência da dengue com as variáveis socioeconômicas do trabalho, tal como se observa na Tabela 2. 
Tabela 2

Correlação entre incidência da dengue com as variáveis selecionadas, por unidades federativas, região Sudeste do Brasil, 2010.

\begin{tabular}{cccccc}
\hline $\begin{array}{c}\text { Unidade } \\
\text { Federativa }\end{array}$ & IDHM & $\begin{array}{c}\text { População } \\
\text { urbana }\end{array}$ & Renda per capita & $\begin{array}{c}\text { Esperança de } \\
\text { vida ao nascer }\end{array}$ & PEA $>$ que 18 anos \\
\hline Rio de Janeiro & 0.343 & 0.081 & 0.222 & 0.166 & -0.061 \\
\cline { 2 - 6 } & 0.000 & 0.440 & 0.033 & 0.113 & 0.562 \\
São Paulo & 0.180 & 0.189 & 0.047 & 0.103 & -0.32 \\
\hline Espírito Santo & 0.000 & 0.000 & 0.228 & 0.009 & 0.408 \\
\hline Minas Gerais & 0.137 & 0.309 & 0.022 & 0.101 & 0.177 \\
\hline
\end{tabular}

Fonte: IBGE (2010) e MS (2010).

Nota: Os valores mostrados nas colunas referem-se respectivamente ao coeficiente de correlação de Pearson (r) e ao nível de significância (valor $p$ ) da correlação.

Para o Estado de Minas Gerais existe associação entre as taxas de infectados por dengue e: IDHM; percentual de população urbana; renda per capita; esperança de vida ao nascer; e população economicamente ativa maior de 18 anos. No entanto, os resultados dos coeficientes de correlação de Parson (r) indicam baixo grau de linearidade positiva. Esse grau é mais elevado $(0,24)$ entre a taxa de incidência de dengue e o grau de urbanização; ou seja, áreas mais urbanizadas estão associadas à maior ocorrência da doença.

No Espírito Santo, houve correlação significativa apenas entre a incidência da dengue e o percentual de população urbana, sendo essa associação linear positiva. Todavia, o coeficiente de correlação $(0,31)$ apresenta grau de associação estatística relativamente baixa entre as variáveis.

No Estado do Rio de Janeiro houve correlação linear positiva entre as variáveis dengue e IDHM; e entre dengue e renda per capita. Mas, nas duas associações, o coeficiente é relativamente baixo.

Em São Paulo o teste de correlação mostra associação entre as taxas da dengue e: IDHM; percentual de população urbana; e esperança de vida ao nascer. Contudo, seguindo a linha dos coeficientes de correlação das outras unidades federativas, também existe pouca associação entre as variáveis.

O uso do IDHM, da renda per capita e da esperança de vida ao nascer nas correlações, pode parecer redundante a princípio. No entanto, conforme os resultados mostrados, quando 
houve associação das taxas de incidência de dengue com o IDHM, não necessariamente houve com as outras variáveis anteriormente citadas, as quais estão inseridas no IDHM (FJP, 2013).

Os resultados obtidos mostram ainda uma correlação linear positiva entre a taxa de incidência de dengue e IDHM nos Estados de Minas Gerais, Rio de Janeiro e São Paulo, quando o esperado seria a correlação negativa, porque quanto maior a incidência da dengue, teoricamente, as condições de vida deveriam ser mais precárias.

O índice IDHM é calculado conforme o acesso ao conhecimento, o direito à vida longa e saudável e a um padrão de existência digno da população (LANA et al., 2009; FISHER et al., 2010). Trata-se, portanto, de uma generalização que busca medir o nível de sucesso atingido por uma sociedade no atendimento às necessidades básicas e universais do ser humano. A correlação positiva entre as variáveis talvez se deva ao fato de, em municípios mais desenvolvidos, geralmente há maior densidade demográfica, e a aglomeração da população pode favorecer a proliferação de focos do mosquito transmissor. Essa mesma explicação pode ser aplicada às correlações positivas observadas entre a incidência de dengue e, às variáveis: renda per capita e esperança de vida ao nascer.

Certas pesquisas apontam para a relação entre a maior aglomeração populacional urbana e a maior incidência da dengue e seu vetor (SANTOS; MARÇAL JÚNIOR, 2004; FONSECA; BRAZ, 2010; BRAZ et al., 2013). Dessa forma, a análise de correlação realizada entre as variáveis: população economicamente ativa, população urbana, e a dengue - foram necessárias para verificar a relação entre a ocorrência de grandes centros urbanos, com seus aglomerados populacionais e áreas de maior atividade produtiva -, com a incidência da doença em análise. Nos dados referentes a Minas Gerais, por exemplo, há correlação significativa $(\mathrm{p}<0.01)$ entre dengue e população urbana, e entre dengue e população economicamente ativa; o que comprova tal relação apontada pela literatura. Mas, esta última correlação pode ser averiguada apenas em Minas Gerais.

Em conformidade com os resultados da análise de correlação deste trabalho, outras pesquisas esclarecem que a dengue é uma doença predominantemente urbana, padrão que é notado em todo o Sul da América (SAWYER; LEITE, 2002; MORENO; BARATA, 2012), mas, na África, também é verificada de forma significativa no meio silvestre (RATMANOV et al., 2013). Enquanto no entorno das cidades o hospedeiro principal do vírus são as pessoas, as quais contaminam os mosquitos disseminadores dessa epidemia; no meio silvestre os principais hospedeiros são macacos, que são potencialmente a fonte de contaminação para si e para humanos, tendo como intermediário principal o mosquito Aedes aegypti. Este vetor, conforme a 
literatura (NEVES, 2005), veio do continente africano para o restante das áreas tropicais do planeta, trazido por meio dos navios negreiros com o tráfico de escravos humanos.

Onde há enfermos com dengue há relação associativa com a ocorrência do vetor de transmissão, o mosquito Aedes aegypti (BARBOSA; LOURENÇO, 2010). Sendo assim, os locais que apresentam altas taxas de incidência de adoentados por dengue, tais como os mostrados, estão mais sujeitos à ocorrência de outras doenças transmitidas pelo mesmo inseto, entre elas: a febre amarela (JENTES et al., 2011), a febre chikungunya (CHAVES et al., 2012) e o zika vírus (CAMPOS et al., 2015).

O emprego do índice de similaridade por vizinhança para cada Estado apresentou resultados significativos $(\mathrm{p}<0,01)$, com Índice Global de Moran igual a 0,502 - conforme é mostrado no Mapa 2. No Estado de Minas Gerais foram observados vinte (20) municípios enquadrados na classe "Muito alta" semelhança por vizinhança; e nove (9) na classe "Alta" semelhança. No Estado de São Paulo foram observados oitenta e quatro (84) municípios enquadrados na classe "Muito alta" semelhança por vizinhança; seis (6) na classe "Alta"; sete (7) na classe

"Baixa"; e noventa e cinco (95) na classe "Muito baixa" semelhança por vizinhança. No Estado do Espírito Santo foram observados três (3) municípios enquadrados na classe "Muito alta" semelhança por vizinhança. No Estado do Rio de Janeiro foram observados dois (2) municípios enquadrados na classe "Muito alta" semelhança por vizinhança. Portanto, as zonas críticas para ocorrência dos surtos de infectados por dengue são aquelas de clusters de cor preta e, em menor grau, as apresentadas de cor vermelha, as quais apresentaram maior interação entre si. 


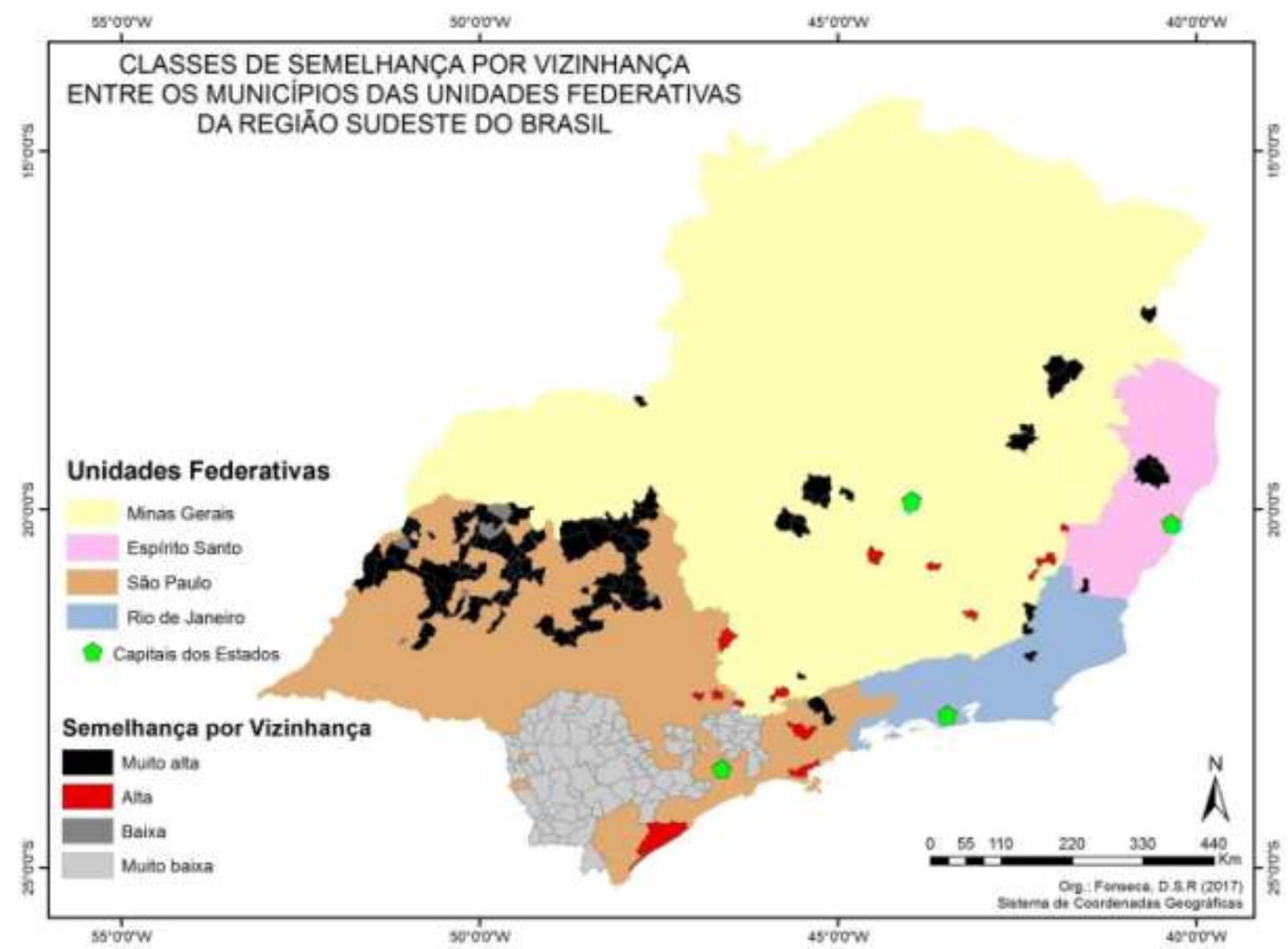

Figura 3. Classes de semelhança por vizinhança, conforme o Índice de Moran, nos municípios com casos notificados de pessoas infectadas por dengue (2010) da região Sudeste do Brasil.

Nos clusters gerados não houve, aparentemente, relação entre a semelhança espacial de infectados por dengue e a proximidade com as capitais dos Estados. Após a geração desses clusters, foi realizada novamente a análise de correlação com todas as variáveis do trabalho, todavia agora, estritamente nos municípios que apresentaram semelhanças por vizinhança nas taxas de infectados por dengue -, os resultados foram muito similares àqueles já observados. As semelhanças espaciais na incidência das taxas de dengue podem estar relacionadas conjuntamente a fatores ambientais, os quais fogem dos objetivos deste trabalho, mas, a partir de tal hipótese, é aberta uma nova possibilidade para pesquisas.

Pode-se afirmar com base nos resultados obtidos que onde se verificou a maior taxa de urbanização associada à ocorrência da dengue (Minas Gerais, São Paulo e Espírito Santo) e essa relação concomitante a renda per capita (Rio de Janeiro e Minas Gerais) - o número de casos de enfermos poderia estar ligado a um processo chamado "deseconomia" (RALFO, 1995), quando os níveis de desenvolvimento atingem um ápice local e, posteriormente, há um processo de aumento da complexidade dos problemas urbanos, com a desconcentração de certas metrópoles e 
fluxo de pessoas para cidades secundárias. Estas cidades, em vários casos, não estão capacitadas para o aumento do movimento migratório e, com isso, ocorre maior propagação de doenças (BIRABEN, 1984).

No caso da epidemia em foco neste trabalho, a contaminação de pessoas saudáveis em áreas distintas pode ocorrer com o hospedeiro humano contaminado, o qual passa o vírus para o mosquito ao ser picado, ou ainda, pelo transporte passivo do vetor, o mosquito Aedes aegypti, grudado no corpo das pessoas que migram (SANTOS; MARÇAL JÚNIOR, 2004). Tal como foi observado na Figura 3, sobretudo em São Paulo e Minas Gerais, onde há regiões "polinucleadas" (LOBO; MATOS, 2011) de ocorrência da dengue, as taxas dessa doença poderiam funcionar como bioindicadoras para um processo de "espraiamento" (AZZONI, 1986) econômico do fluxo de capital e pessoas?

\section{4- CONSIDERAÇÕES FINAIS}

A unidade federativa do Sudeste que apresentou a maior taxa média de incidência de dengue foi o Rio de Janeiro (média 280.198), seguida por São Paulo (média 204.087). Minas Gerais (média 159.386) foi o Estado com a menor incidência, seguido pelo Espírito Santo (média 183.949).

Houve correlação entre dengue e IDHM nas seguintes unidades federativas: Minas Gerais, Rio de Janeiro e São Paulo. Entre dengue e percentual de população urbana: em Minas Gerais, Espírito Santo e São Paulo. Entre dengue e renda per capita: em Minas e no Rio de Janeiro. Entre dengue e esperança de vida ao nascer: em Minas e São Paulo. A associação entre dengue e população economicamente ativa foi verificada somente no Estado de Minas Gerais.

A análise espacial por semelhança de vizinhança com as taxas de dengue mostrou clusters localizados de forma heterogênea para o Estado de Minas Gerais, no entanto, houve maior homogeneidade em São Paulo, Rio de Janeiro e Espírito Santo, são essas as áreas críticas, com maior prevalência de enfermos. Essa maior dispersão nos dados foi verificada também no coeficiente de variação dessas mesmas taxas para Minas Gerais (144,03\%), o que pode estar condicionado a outros fatores passíveis de correlação com a incidência da dengue, tais como aqueles de ordem ambiental, os quais fogem aos objetivos deste trabalho, mas cria possibilidades para novas pesquisas.

Os resultados da análise de componentes principais e das correlações, especialmente referente àquilo que foi verificado entre: incidência da dengue e IDHM, dengue e renda per capita 
- mostraram não existir relação direta entre o nível de desenvolvimento das sociedades e a inibição de desordens epidemiológicas coletivas. Tem ocorrido, tal como mostrado, o contrário, quanto maior o nível de desenvolvimento, maior é a possibilidade da ocorrência de dengue e, menor tem sido a capacidade de tais sociedades em conter tal distúrbio da saúde coletiva.

\section{REFERÊNCIAS BIBLIOGRÁFICAS}

AZZONI, C. Indústria e reversão da polarização no Brasil. Ensaios Econômicos, São Paulo, IPE/USP, n. 58, 1986.

ANSELIN, L. Local Indicators of Spatial Association-LISA. Geographical Analysis, v. 27, n.2, p. 93-115, 1995.

AYRES, M. BioEstat. Versão 5.0. Soc. Civ. Mamirauá, MCT - CNPq. Belém: 2007, 290p.

BARBOSA, G. L.; LOURENÇO, R. W. Análise da distribuição espaço-temporal de dengue e da infestação larvária no município de Tupã, Estado de São Paulo. Revista da Sociedade Brasileira de Medicina Tropical, v. 43, n. 2, p. 145-151, 2010.

BIRABEN, Jean-Nöel. Epidemias na História da População. In: MARCÍLIO, Maria L. (org.) População e Sociedade. Petrópolis: Vozes, 1984, p. 111-131.

BRAZ, C. K. R.; FONSECA, D. S. R.; AMARAL, V.B.; MIRANDA, W. A. Teste da correlação entre hipsometria e índices de ocorrência do mosquito Aedes aegypti na cidade de Montes ClarosMG com dados dos anos 2009 e 2010. Caminhos de Geografia, v. 14, n. 47, p. 199-207, 2013.

CAMPOS, G. S; BANDEIRA, A. C.; SARDI, S. I. Zika virus outbreak, Bahia, Brazil. Emerging Infectious Diseases, v. 21 n. 10, p. 1885-1886, 2015.

CAMPOS, M.B.; MACEDO, D. R. Agrupamentos de emigração internacional no Brasil: o papel das redes sociais na formação dos espaços de emigração. GEOGRAFIA, v. 39, n. 2, p. 257-272, 2014.

CARVALHO, L. E. et al. Regressão Linear Geograficamente Ponderada em ambiente SIG. Transportes, v. 14, n. 2, p. 18-26, 2006.

CARVALHO, J. R. P; ASSAD, E. D.; PINTO, H. S. Interpoladores geoestatísticos na análise da distribuição espacial da precipitação anual e de sua relação com altitude. Pesquisa Agropecuária Brasileira, v. 47, n. 9, p. 1235-1242, 2012.

CASTRO, F. S; PEZZOPANE, J. E. M; CECILIO, R. A; PEZZOPANE, J. R. M; XAVIER, A. C. Avaliação do desempenho dos diferentes métodos de interpoladores para parâmetros do balanço hídrico climatológico. Revista Brasileira de Engenharia Agrícola e Ambiental, v. 14, n. 8, p. 871-880, 2010. 
CHAVES, T. S. S; PELLINI, A. C. G; MASCHERETTI, M; JAHNEL, M. T; RIBEIRO, A. F; RODRIGUES, S. G; VASCONCELOS, P. F. C.; BOULOS, M. Travelers as sentinels for Chikungunya Fever, Brazil. Emerging Infectious Diseases, v. 18, n. 3, p. 529-530, 2012.

FONSECA, D. S. R.; BRAZ, C. K. R. Investigação epidemiológica da distribuição geográfica do Aedes aegypti na cidade de Montes Claros (MG) com uso de geotecnologias. Revista Brasileira de Cartografia, v. 62, n. 3, p. 489-503, 2010.

FJP, FUNDAÇÃO JOÃO PINHEIRO. Atlas do Desenvolvimento Humano - 2013. Disponível em: $\quad$ http://www.fjp.mg.gov.br/index.php/produtos-e-servicos1/2610-indicededesenvolvimento-humano-idh-2. Acessado em 29 ago. de 2017.

IBGE, INSTITUTO BRASILEIRO DE GEOGRAFIA E ESTATÍSTICA. Resultados: censo 2010. Disponível em: <https://censo2010.ibge.gov.br/resultados.html>. Acessado em 30 ago. de 2017.

IBGE, INSTITUTO BRASILEIRO DE GEOGRAFIA E ESTATÍSTICA. Bases e referenciais. Disponível em: <https://mapas.ibge.gov.br/bases-e-referenciais/basescartograficas/malhasdigitais.html>. Acessado em 30 ago. de 2017.

IPEA, INSTITUTO DE PESQUISAS ECONÔMICAS APLICADAS. Secretaria de Assuntos Estratégicos. IPEAGEO: Bases. Disponível em:

<http://www.ipea.gov.br/ipeageo/bases.html>. Acessado em: 30 ago. de 2017.

JENTES, E. S.; POUMEROL, G.; GERSHMAN, M. D.; HILL, D. R.; LEMARCHAND, J.; LEWIS, R. F.; STAPLES, J. E.; TOMORI, O.; SMITH, A. W.; MONATH, T. P. The revised global yellow fever risk map and recommendations for vaccination, 2010: consensus of the Informal WHO Working Group on Geographic Risk for Yellow Fever. The Lancet, v. 11, p. 622632, 2011.

LANA, F. C. F; DAVI, R. F. L; LANZA, F. M.; AMARAL, E. P. Detecção da hanseníase e Índice de Desenvolvimento Humano dos municípios de Minas Gerais, Brasil. Revista Eletrônica de Enfermagem, v. 11, n. 3, p. 539-544, 2009.

LOBO, C.; MATOS, R. Migrações e a dispersão espacial da população nas Regiões de Influência das principais metrópoles brasileiras. Revista Brasileira de Estudos Populacionais, v. 28, n. 1, p. 81-101, 2011.

MATOS, R. Questões teóricas acerca dos processos de concentração e desconcentração da população no espaço.Revista Brasileira de Estudos Populacionais, v. 12, n. 2, p. 35-58, 1995.

MARTINS, G. A. Estatística geral e plicada. São Paulo: Atlas, 2010. 
MS, MINISTÉRIO DA SAÚDE. Portal da Saúde. Acesso à Informação. Taxa de incidência de dengue, período 2010-2011. Disponível:

http://portalsaude.saude.gov.br/index.php/acesso-a-informacao $>$. Acessado em: 30 jan. de 2016.

MORENO, E. S.; BARATA, R. C. B. Methodology for definition of yellow fever priority areas, based on environmental variables and multiple correspondence analyses. PLoS Biology, v. 6, n. 7, p. 1-7, 2012.

NEVES, D. P. Parasitologia humana. São Paulo: Atheneu, 2005.

PESSANHA, J. E. M; CAIAFFA, W. T; CÉSAR, C. C.; PROIETTI, F. A. Avaliação do Plano Nacional de Controle da Dengue. Caderno de Saúde Pública, v. 25, n. 7, p. 16371641, 2009.

PIETRI, D. E.; GARCÍA, S.; RICO, O. Modelos geo-espaciales para la vigilancia local de la salud. Revista Panamericana de Salud Pública, v. 23, n. 3, p. 394-402, 2008.

RATMANOV, P.; MEDIANNIKOV, O.; RAOULT, D. Vector borne diseases in West Africa: geographic distribution and geospatial characteristics. Transactions of the Royal Society of Tropical Medicine and Hygiene, v. 107, p. 273-284, 2013.

REGIS, L.; SOUZA, W. V.; FURTADO, A. F.; FONSECA, C. D.; SILVEIRA JÚNIOR, J. C.; RIBEIRO JÚNIOR, P. J.; SANTOS, M. A. V. M.; CARVALHO, M. S.; MONTEIRO, A. M. V. An entomological surveillance system based on open spatial information for participative dengue control. Anais da Academia Brasileira de Ciências, v. 81, n. 4, p. 655662, 2009.

SANTOS, A.; MARÇAL JÚNIOR, O. Geografia do dengue em Uberlândia (MG) na epidemia de 1999. Caminhos de Geografia, v. 3, n. 11, p. 35-52, 2004.

SAWYER, D. O.; LEITE, I. C.; ALEXANDRINO, R. A. Perfis de utilização de serviços de saúde no Brasil. Ciência \& Saúde Coletiva, v. 7, n. 4, p. 757-776, 2002.

SILVA, W. L.; DERECZYNSKI, C. P. Caracterização climatológica e tendências observadas em extremos climáticos no Estado do Rio de Janeiro. Anuário do Instituto de Geociências: UFRJ, v. 37, n. 2, p. 123-138, 2014.

VIOLA, M. R; MELLO, C. R; PINTO, D. B. F.; ÁVILA, L. F. Métodos de interpolação espacial para o mapeamento da precipitação pluvial. Revista Brasileira de Engenharia Agrícola e Ambiental, v. 14, n. 9, p. 970-978, 2010. 